\title{
Outcomes when Cancer Treatment is Delivered on, or off, a Clinical Trial: Implications for Generalizability of Study Results
}

\author{
Maurie Markman \\ Cancer Treatment Centers of America, Eastern Regional Medical Center, Philadelphia, Pa., USA
}

There has been considerable discussion in the oncology literature regarding the generalizability of the results of clinical trials to the population of individuals with a particular condition who either would have been 'poor candidates' for treatment (e.g. borderline performance status) or would have been specifically excluded from study participation (e.g. co-morbid medical condition) [1]. Obvious questions that result from this concern include the therapeutic benefits anticipated to be observed in these patients and possible increased morbidity that might be experienced by individuals who would otherwise not have been eligible for study-based treatment.

In an attempt to at least indirectly examine the extent of differences versus similarities between populations included in versus those likely excluded from clinical trials, investigators have explored their unique experiences in this arena.

One such analysis, reported by Yang et al. [2] from the National Taiwan University Hospital and National Taiwan University College of Medicine (Taipei, Taiwan) in this issue of Oncology examined the survival of 159 individuals among a total population of 1,475 patients with cancer of the pancreas treated from 1995 through
June 2007 who received a gemcitabine-based chemotherapy program. Within this group, 30 patients were treated on a trial, and 129 were not entered into a clinical study.

As is the case with all retrospective evaluations of nonrandomized populations there are clearly recognized and unrecognized imbalances between subgroups, based on the reasonable clinical judgment employed by the individual treating physicians dealing with specific patients. However, a particular advantage in the current analysis is that all included patients had to possess an acceptable clinical status (and absence of co-morbidities that would preclude such therapy) to at least be a candidate for treatment with gemcitabine.

A major point to be made in analyzing the data included in this report is that a substantially higher percentage of patients who were not treated on a clinical trial initiated therapy with a marginal performance status (level 3; 37 of 129 patients), compared to those individuals entered to a study ( 0 of 30 patients). Therefore, perhaps not surprisingly, the patient population treated on a clinical trial experienced substantially superior survival (median: 14.4 months versus 4.9 months; $\mathrm{p}<0.0001$ ). Yet, this is a relevant real-world experience, and the data suggest

\section{KARGER}

Fax +41613061234 E-Mail karger@karger.ch www.karger.com
(ㄷ) 2011 S. Karger AG, Basel

0030-2414/11/0814-0141\$38.00/0

Accessible online at:

www.karger.com/ocl
Maurie Markman, MD

Cancer Treatment Centers of America, Eastern Regional Medical Center

1331 East Wyoming Avenue

Philadelphia, PA 19124 (USA)

Tel. +1 215537 7502, E-Mail maurie.markman@ctca-hope.com 
the magnitude of the impact associated with this single critical factor on both patient selection for entry into clinical trials [3], and on the ultimate outcome of the combined effect of therapy and the natural history of this devastating malignancy.

Of interest, a recent report from Japan exploring a similar question in patients with gastric cancer did not observe an impact on overall survival associated with trial-based treatment [4]. The difference in outcomes in these two reasonable attempts to examine a highly relevant clinical question emphasizes the complexity of the topic and how difficult it is to draw universal conclusions.
For the present, it is reasonable to conclude that patients who participate in clinical trials may possess more favorable clinical characteristics compared to individuals who elect to not participate, are not offered participation, or are specifically excluded from trial entry due to an unacceptable performance status or co-morbid medical conditions [3]. How oncologists might optimally apply well-conceived study results outside the confines of often quite homogenous clinical trial populations to the much larger and highly heterogeneous group of individuals with a particular malignant condition should be a matter for considerable future discussion and debate.
References
Elting LS, Cooksley C, Bekele BN, et al: Generalizability of cancer clinical trial results: prognostic differences between participants and nonparticipants. Cancer 2006; 106 2452-2458.

-2 Yang SH, Kuo YH, Tien YW, et al: Inferior survival of advanced pancreatic cancer patients who received gemcitabine-based chemotherapy but did not participate in clinical trials. Oncology 2011;81:143-150.
3 Conroy T, Desseigne F, Ychou M, et al: FOLFIRINOX versus gemcitabine for metastatic pancreatic cancer. N Engl J Med 2011;364: 1817-1825.

4 Tanai C, Nakajima TE, Nagashima K, et al: Characteristics and outcomes of patients with advanced gastric cancer who declined to participate in a randomized clinical chemotherapy trial. J Oncol Practice 2011;7: 148-153. 